\title{
Detrimental impact of contagious disease cues on consumer preference for anthropomorphic products
}

\author{
Ying Ding ${ }^{1} \cdot$ Sunxu $X^{1}{ }^{1}$
}

Accepted: 4 January 2022 /Published online: 17 January 2022

(C) The Author(s), under exclusive licence to Springer Science+Business Media, LLC, part of Springer Nature 2022

\begin{abstract}
Despite the extensive use of anthropomorphism strategy in marketing practices, little research attention has been given to the environmental factors that influence consumer preference for anthropomorphic products. This research examines when and why contagious disease cues can influence consumer preference for anthropomorphic products. The results from four empirical experiments consistently show that when exposed to contagious disease cues, consumers exhibit a lower preference for anthropomorphic products (Study 1), which is mediated by social withdrawal (Study 2). Furthermore, our findings demonstrate that this detrimental effect would be attenuated for products in digital (vs. physical) format (Study 3), or in regions with low (vs. high) local severity of the contagious disease (Study 4). These findings contribute to the literature on contagious diseases and anthropomorphism and offer important managerial implications.
\end{abstract}

Keywords Contagious disease cues $\cdot$ Anthropomorphic products $\cdot$ Social withdrawal $\cdot$ Product format $\cdot$ Local severity of the contagious disease

\section{Introduction}

Anthropomorphism refers to the attribution of human characteristics to non-human entities (Epley et al., 2007), which has been widely used in marketing, for example, constructing a human-like brand mascot (e.g., Michelin Man), selecting a human

\footnotetext{
Ying Ding and Sunxu Xu contributed equally, and the order of authorship is alphabetically determined.

Sunxu Xu

xusunxu@ruc.edu.cn

Ying Ding

dingying@rmbs.ruc.edu.cn

1 Renmin Business School, Renmin University of China, No. 59 Zhonguancun Street, Beijing 100872, People's Republic of China
} 
brand name (e.g., Mr. Muscle), and designing products with human facial or physical features (e.g., Beats Pill 2.0 speaker). Prior research mainly demonstrates that anthropomorphism could generate more favorable attitudes toward products and enhance consumer-brand connections (Aggarwal \& McGill, 2007; Fournier, 1998), implying that anthropomorphic products are more attractive in general.

However, little research has concentrated on anthropomorphism's detrimental effects. Recent research focusing on this question found anthropomorphism may backfire for consumers in socially crowded contexts or under high distinctiveness motives (Puzakova \& Aggarwal, 2018; Puzakova \& Kwak, 2017). Although these studies provide meaningful insights, the effects of environment-related factors such as contagious disease cues on consumers' preference for anthropomorphic products remain unclear.

Since its outbreak, COVID-19 has spread worldwide and caused enormous social changes (Sheth, 2020). In this study, we aim to extend its effect to product preference and investigate when and why contagious disease cues can affect consumer preference for anthropomorphic products. Building on behavioral immune system (BIS) literature, the activation of BIS when faced with contagious diseases can elicit greater social withdrawal (Mortensen et al., 2010), which, we posit, would reduce preference for anthropomorphic products. Further, we argue that this detrimental effect would be attenuated for products in digital (vs. physical) format, or in regions with low (vs. high) local severity of the contagious disease.

We test these hypotheses across a set of four experiments using multiple manipulation methods and real choice settings. These findings contribute to the literature on both contagious disease and anthropomorphism and provide meaningful implications for marketing practice.

\section{Theoretical background}

\subsection{Effect of contagious disease cues on preference for anthropomorphic products}

Contagious disease cues are information related to the spread of contagious diseases that can evoke concerns about being infected (Galoni et al., 2020), for example, news about COVID-19. Accordingly, there are two key characteristics of contagious disease cues: vulnerability to the contagious disease and human-to-human transmission (Huang \& Sengupta, 2020). When feeling vulnerable to contagious diseases, given that contagious diseases are commonly transmitted through interpersonal interaction, the activated BIS would instinctively lead individuals to exhibit heightened avoidant behaviors when confronted with human faces (Mortensen et al., 2010). Similarly, infection-related cues would reduce relative preference for products that are conceptually associated with more consumers (Huang \& Sengupta, 2020). Therefore, when interpersonal interaction is salient, we propose that contagious disease cues would facilitate social withdrawal, including avoidance of interpersonal contact (Baum \& Koman, 1976; Puzakova \& Kwak, 2017). 
Since consumers can establish interpersonal relationships with anthropomorphic products and obtain quasi-social experiences, anthropomorphism can serve as a salient cue for interpersonal interaction (Chen et al., 2017; Fournier, 1998). Due to the definition of anthropomorphism, when faced with objects in anthropomorphic terms, individuals tend to apply social attitudes or beliefs to the inanimate world (Chen et al., 2017; Kim \& McGill, 2018). For example, in assessing the appropriateness of anthropomorphic brand behaviors, consumers tend to use the same standards as humans (Boeuf, 2020). When exposed to contagious disease cues, individuals would instinctively avoid unnecessary interpersonal interactions because others may carry the virus (Mortensen et al., 2010). Therefore, social withdrawal evoked by contagious disease cues could extend to anthropomorphic products and lead to reduced preference. Accordingly, we hypothesize:

H1. Exposure to contagious disease cues (vs. no exposure) will decrease consumer preference for anthropomorphic products.

H2. The effect of contagious disease cues on consumer preference for anthropomorphic products will be mediated by social withdrawal.

\subsection{Boundary conditions}

Given the two key characteristics of contagious disease cues, we further examine two boundary conditions of the proposed detrimental effect.

\subsubsection{Product format}

Digital products refer to intangible goods that can be stored, delivered, and used in electronic format, which are proliferating widely in the increasingly liquid world (Belk, 2013). Since physical contact is the premise of human-to-human transmission of disease, we argue that the contagious disease cues effect is more likely to manifest for anthropomorphic products in physical format. Conversely, there is usually no physical contact involved in digital products. When anthropomorphic products exist in digital format, consumers would not view them as potential sources of disease infection and thus alleviate the detrimental effect. Hence, we hypothesize:

H3. The effect of contagious disease cues on consumer preference for anthropomorphic products will be moderated by product format. Specifically, the detrimental effect will be attenuated for anthropomorphic products in digital format.

\subsubsection{Local severity of the contagious disease}

In this research, local severity of the contagious disease refers to the current spread severity of the contagious disease in the region where the target consumers reside, which serves as a proxy for perceived vulnerability to the contagious disease, one of the key characteristics of contagious disease cues. The World Health Organization's 
weekly epidemiological update reports indicate that local severity is highly heterogeneous as COVID-19 continues to spread (WHO, 2021). Therefore, even in the same period, perceived vulnerability would differ significantly in regions with different severity levels, which would moderate disease-avoidant psychological responses (Schaller \& Murray, 2008). The high level of perceived vulnerability for consumers in high-severity regions would result in the proposed detrimental effect. By contrast, consumers in low-severity regions are not at high risk of infection, and the relatively low level of perceived vulnerability might mitigate the detrimental effect. Hence, we hypothesize:

H4. The effect of contagious disease cues on consumer preference for anthropomorphic products will be moderated by local severity of the contagious disease. Specifically, the detrimental effect will be attenuated for consumers in low-severity regions.

\section{Research method}

\subsection{Study 1: Preliminary evidence of the detrimental effect}

Study 1 aimed to provide initial evidence to support the detrimental effect of contagious disease cues (H1). We also included an accident threat condition to demonstrate that if the threat is not related to interpersonal interaction, this effect will be mitigated.

\subsubsection{Method}

Study 1 adopted a 3 (contagious disease cue vs. accident cue vs. control) betweensubjects design. We recruited participants from Credamo, a leading online platform for experiments in China, and received 229 valid responses $(56.3 \%$ female; $M_{\text {age }}=29.28$ ).

First, all participants were randomly assigned to read one of the following three news reports adapted from Liu and Smeesters (2010) and Huang et al. (2018): COVID-19 (contagious disease condition), air crashes (accident condition), and dental technique (control condition). These news reports were sourced from real information and were approximately the same length. After reading them, we asked participants to summarize the news and describe their thoughts. We then measured the perceived quality of the news $(1=$ very uninformative to $7=$ very informative).

Next, all participants were instructed to imagine they were considering purchasing a storage box and had two options. We manipulated the degree of anthropomorphism by changing the arrangement of rectangles on the front of the box (Kim \& McGill, 2011). For the low-anthropomorphic option, we connected three rectangles as a single line (see appendix). For the high-anthropomorphic option, we arranged 
three rectangles to form human facial features. Then, participants were asked to indicate their preference between the two options on an 8-point scale.

\subsubsection{Results}

Since the relative preferences were negatively skewed, participants' relative preferences were reverse-coded and subjected to a log transformation (May \& Irmak, 2014), with a higher value indicating a lower preference for anthropomorphic products. As predicted, there was no significant difference between the accident $(M=1.05, S D=0.80)$ and the control condition $(M=1.03, S D=0.87, F(1$, $148)=0.01, p=0.91)$. We then combined these two conditions, and the result showed that participants in the contagious disease condition reported a lower preference for anthropomorphic products $(M=1.31, S D=0.74, F(1,227)=6.12, p=0.01$, $\left.\eta_{P}^{2}=0.03\right)$ compared with the other two conditions $(M=1.04, S D=0.83)$. We also analyzed the news report quality to rule out its potential influence. No significant differences were found across the conditions, and results were identical if we included this variable in the analysis.

\subsection{Study 2: Mediating role of social withdrawal}

Study 2 tested the robustness of the main effect by using a real choice and validated the mediating role of social withdrawal. Additionally, considering the negative and threatening characteristics of contagious disease cues, we measured participants' mood to rule out its potential influence.

\subsubsection{Method}

Study 2 employed a 2 (contagious disease cues: salient vs. control) $\times 2$ (anthropomorphic design: high vs. low) between-subjects design. Participants were recruited from Credamo, and 218 valid responses were received ( $49.1 \%$ female; $\left.M_{\text {age }}=28.71\right)$.

After receiving instructions, all participants were assigned to a reading task similar to Study 1. They were then asked to indicate their mood on a two-item 7-point scale (valence: $1=$ very negative to $7=$ very positive; arousal: $1=$ very calm to $7=$ very arousing). Next, participants were presented with a volunteer recruitment advertisement from a national environmental non-governmental organization, the Green Resources Association. In both conditions, the brochure contents were the same, but we manipulated the degree of anthropomorphism by changing the association logo and slogan (see appendix). In the low-anthropomorphic condition, we used only the recycling symbol as the association logo, and the slogan read "Join the association to build a beautiful homeland!" In the high-anthropomorphic condition, we added hands and feet to the recycling symbol, and the slogan read "Join me to build a beautiful homeland!" After reading the brochure, participants were asked to indicate whether they would join the association and participate in future voluntary activities in their neighborhoods. Participants who chose to join the association were required to provide their phone numbers and email addresses for contact. 
Next, social withdrawal was measured using three items adapted from Leary et al. (2003) and Puzakova and Kwak (2017) on 7-point scales ranging from 1=strongly disagree to $7=$ strongly agree (e.g., At this moment, I would like to keep my distance with others; Cronbach's $\alpha=0.86$ ). Finally, participants were asked to indicate the anthropomorphic and aesthetic perception of the logo design on 7-point scales ( $1=$ very low to $7=$ very high).

\subsubsection{Results}

Manipulation check As predicted, participants rated the logo in the high-anthropomorphic condition as more anthropomorphic $(M=5.78, S D=1.26)$ than that in the low-anthropomorphic condition $(M=4.57, S D=1.78, F(1,216)=33.15, p<0.001$, $\left.\eta_{P}^{2}=0.13\right)$. Additionally, no significant difference was found between the two conditions in terms of the aesthetic perception $\left(M_{\text {high-anthropomorphic }}=5.56, S D=1.53\right.$ vs. $\left.M_{\text {low-anthropomorphic }}=5.81, S D=1.22 ; F(1,216)=1.83, p=0.18\right)$.

Participation rate Regressing the participation choice $(1=$ join, $0=$ not join $)$ on contagious disease cues $(1=$ salient, $0=$ control), anthropomorphic design $(1=$ high, $0=$ low), and their interaction in a logistic regression yielded a significant interaction effect $(\beta=-2.73$, Wald $\chi 2=14.22, p<0.001$; odds ratio $=0.07)$ and a significant main effect of contagious disease cues $(\beta=1.26$, Wald $\chi 2=4.96, p=0.03$; odds ratio=3.53). Supporting $\mathrm{H} 1$, in the high-anthropomorphic condition, a smaller proportion of participants were willing to join the association after exposure to contagious disease cues (53.06\%) compared to those not exposed to disease cues $(83.05 \%, \beta=-1.47$, Wald $\chi 2=10.63, p<0.01$; odds ratio $=0.23$; see Fig. 1). In the low-anthropomorphic condition, participants in the contagious disease condition $(91.38 \%)$ had a higher participation rate than those in the control condition $(75.00 \%, \beta=1.26$, Wald $\chi 2=4.96, p=0.03$; odds ratio $=3.53)$. This may be because contagious disease cues can elicit mortality salience, thereby increasing individuals' sense of belongingness to the association that supports their worldview. However, this potential positive influence was diminished when the high-anthropomorphic

Fig. 1 Impact of contagious disease cues on participation rate (Study 2)

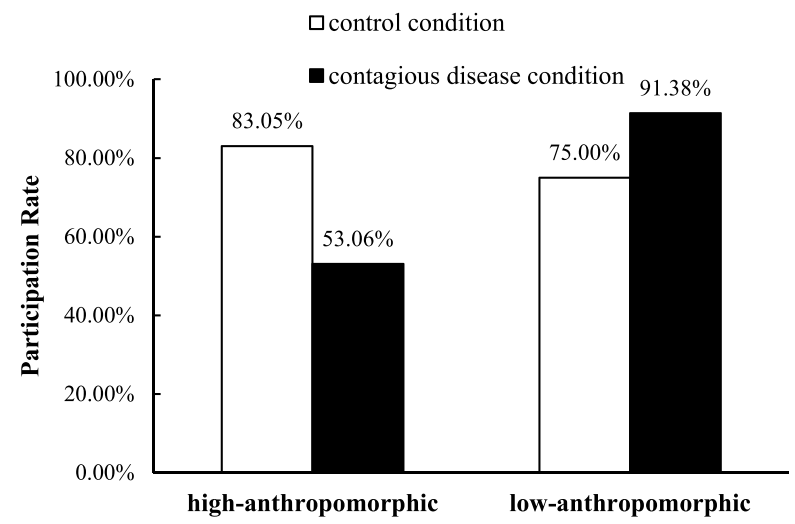


design reminds the infection risk through interpersonal interactions (for more details, please see the posttest results in web appendix).

Mediation Analysis The results of a $2 \times 2$ ANOVA on social withdrawal indicated a significant main effect of contagious disease cues $\left(F(1,214)=18.50, p<0.001, \eta_{P}^{2}\right.$ $=0.08$ ) and a significant interaction between contagious disease cues and anthropomorphic design $\left(F(1,214)=8.93, p<0.01, \eta_{P}^{2}=0.04\right)$. Since the high-anthropomorphic design can serve as a salient reminder of potential interpersonal interactions, participants in the contagious disease condition reported higher social withdrawal than those in the control condition when the design was high-anthropomorphic $\left(M_{\text {salient }}=4.08, S D=1.62\right.$ vs. $M_{\text {control }}=2.66, S D=1.31 ; F(1,214)=26.26, p<0.001$, $\left.\eta_{P}^{2}=0.11\right)$. When the design was low-anthropomorphic, no significant difference was found between the conditions $\left(M_{\text {salient }}=3.44, S D=1.49\right.$ vs. $M_{\text {control }}=3.19$, $S D=1.34 ; F(1,214)=0.87, p=0.35)$. We then performed a moderated mediation analysis using the PROCESS SPSS macro (model 8; Hayes, 2013) with 5000 bootstrapped samples. Notably, the $95 \%$ confidence interval for the indirect effect of social withdrawal did not include zero (index $=-0.31,95 \% \mathrm{CI}=-0.84$ to -0.04 ), supporting H2. The conditional effect of contagious disease cues on participation choice was significantly mediated by social withdrawal in the high-anthropomorphic condition (effect size $=-0.38,95 \% \mathrm{CI}=-0.85$ to -0.06 ) but not in the low-anthropomorphic condition (effect size $=-0.07,95 \% \mathrm{CI}=-0.31$ to 0.05 ). The results were identical if we included the mood valence and arousal in the model, ruling out mood as an alternative explanation.

\subsection{Study 3: Moderating role of product format}

In this study, we aimed to further validate the mechanism underlying the contagious disease cues effect by examining the moderating role of product format.

\subsubsection{Method}

Study 3 employed a 2 (contagious disease cues: salient vs. control) $\times 2$ (product format: digital vs. physical) between-subjects design. Participants were recruited from Credamo, and 192 valid responses were received ( $49.5 \%$ female; $M_{\text {age }}=27.66$ ).

First, all participants were instructed to read a popular science article adapted from Liu and Smeesters (2010). To enhance credibility, they were told that the article came from Guokr.com, a popular science website in China. In the article, scientists either predicted that humans will become extinct because of super infectious diseases or lose healthy teeth because of increasingly refined diets. Participants were then instructed to summarize the article and describe their thoughts.

Next, participants were instructed to imagine they were about to receive a membership discount card for the GEONO store, either in digital or physical format. Then, participants were asked to browse two membership card designs, which differed in the degree of anthropomorphism (see appendix). We instructed 
the participants to indicate their preference for each design on 7-point scales ( $1=$ not like it at all to $7=$ like it very much).

After then, participants were asked to complete the same social withdrawal measures as in Study 2. Given that the articles were fictional, we also measured the perceived familiarity of Guokr.com ( $1=$ very unfamiliar to $7=$ very familiar $)$ and the perceived credibility of the article $(1=$ very incredible to $7=$ very credible). Finally, participants evaluated the degree of anthropomorphism of the two card designs.

\subsubsection{Results}

Manipulation check The results from the paired-samples t-test showed that the anthropomorphic perception of membership cards $\left(M_{\text {high-anthropomorphic }}=5.38\right.$, $S D=1.26, M_{\text {low-anthropomorphic }}=3.48, S D=1.52, t(191)=12.10, p<0.001, d=1.36$ ) was manipulated successfully.

Product preference Following Huang and Sengupta (2020), we first computed a difference score by subtracting preference for the low-anthropomorphic design from preference for the high-anthropomorphic design. As in Study 1, the difference score was reverse-coded and subjected to a log transformation to correct for negative skewness, with a higher number indicating a lower relative preference for anthropomorphic products. Compared with participants in the control condition, those in the contagious disease condition were marginally less familiar with Guokr.com $\left(M_{\text {salient }}=4.52, S D=1.45\right.$ vs. $M_{\text {control }}=4.87, S D=1.38 ; t(190)=1.73$, $p=0.09, d=0.25)$ and rated the article as less credible $\left(M_{\text {salient }}=4.99, S D=1.17\right.$ vs. $\left.M_{\text {control }}=5.43, S D=1.09 ; t(190)=2.73, p<0.01, d=0.39\right)$. Therefore, we included these two variables in the following analysis as control variables. A $2 \times 2$ ANCOVA on the difference score yielded only a significant interaction effect $(F(1,186)=6.75$, $\left.p=0.01, \eta_{P}^{2}=0.04\right)$. In line with $\mathrm{H} 3$, when the membership card was in physical format, participants in the contagious disease condition reported a lower relative preference for the high-anthropomorphic design than those in the control condition $\left(M_{\text {salient }}=1.62, S D=0.42\right.$ vs. $M_{\text {control }}=1.38, S D=0.49 ; F(1,186)=4.39, p=0.04$, $\eta_{P}^{2}=0.02$ ). When the membership card was in digital format, however, no significant difference was found between the conditions $\left(M_{\text {salient }}=1.29, S D=0.55\right.$ vs. $\left.M_{\text {control }}=1.47, S D=0.62 ; F(1,186)=2.36, p=0.13\right)$.

Mediation analysis The results of a $2 \times 2$ ANCOVA on social withdrawal indicated only a significant main effect of contagious disease cues $\left(M_{\text {salient }}=3.18, S D=1.07\right.$ vs. $\left.M_{\text {control }}=2.76, S D=1.26 ; F(1,186)=4.07, p<0.05, \eta_{P}^{2}=0.02\right)$. We then performed a moderated mediation analysis using the PROCESS SPSS macro (model 14; Hayes, 2013) with 5000 bootstrapped samples (see Fig. 2). The 95\% confidence interval for the indirect effect of social withdrawal did not include zero (index $=-0.06,95 \% \mathrm{CI}=-0.20$ to -0.00 ). Specifically, the conditional effect of contagious disease cues on the difference score for the anthropomorphic design was 


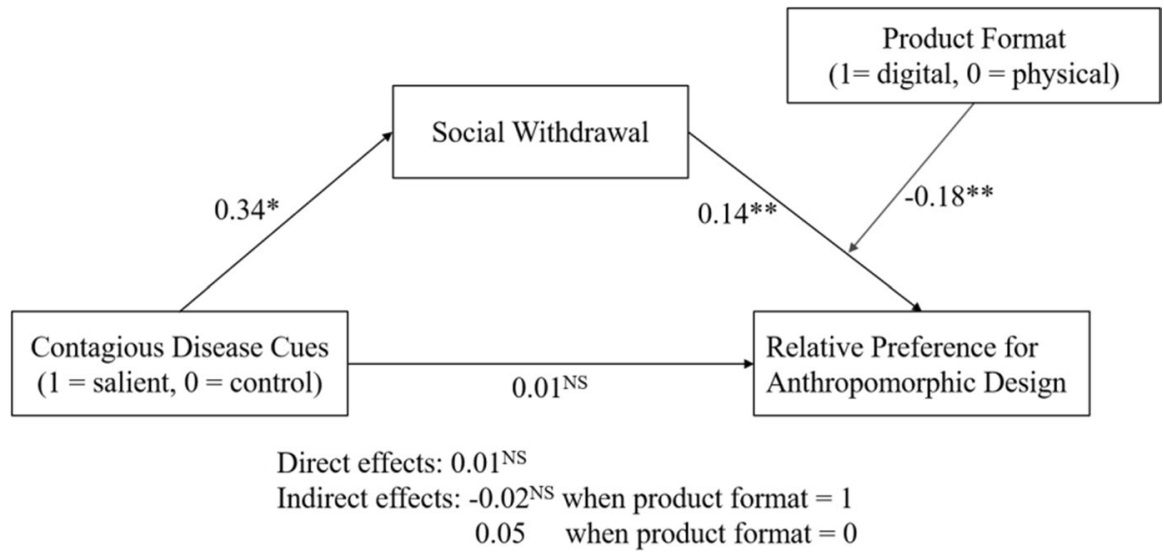

Fig. 2 Moderated mediation model (Study 3)

significantly mediated by social withdrawal in the physical format condition (effect size $=0.05,95 \% \mathrm{CI}=0.00$ to 0.13 ), but not in the digital format condition (effect size $=-0.02,95 \% \mathrm{CI}=-0.11$ to 0.02$)$.

\subsection{Study 4: Moderating role of local severity of the contagious disease}

In Study 4, we aimed to investigate whether the proposed effect would vary according to different levels of perceived vulnerability to the contagious disease by using local severity of the contagious disease as a proxy variable. This study was conducted on Prolific, a leading online participant recruiting platform with a diverse population. In the final sample, the participants were from more than 20 different countries (e.g., UK, USA, Australia, etc.). To capture the current spread severity of the contagious disease in each country, we then collected COVID-19 data from the 39th edition of the World Health Organization's weekly epidemiological update reports, which was the most recent data when Study 4 was completed (WHO, 2021). This report provided the number of "new cases in last 7 days" for each country, and we divided these by the population data to obtain the number of "new cases per 100,000 population" for each country as the local severity index, which ranged from 0.37 to 295.53. We also manipulated the presence of a reminder of quarantine to explore a possibility that when people received too much social isolation, the negative effect of contagious disease cues on preference for anthropomorphic products may be attenuated due to the need for social closeness.

\subsubsection{Method}

In total, 257 valid responses were collected (39.3\% female; $M_{\text {age }}=27.47$ ). All the participants were randomly assigned to the contagious disease cue with a reminder of quarantine condition, the contagious disease cue without a reminder of quarantine condition, or the control condition. 
The manipulation of contagious disease cues was similar to Study 1. Subsequently, participants in the contagious disease cue with a reminder of quarantine condition were instructed to imagine that that day was their $8^{\text {th }}$ day of quarantine in an isolation ward because they were confirmed as a close contact of a COVID19-positive patient. They were instructed to describe their feelings and thoughts about the quarantine. Participants in the other two conditions did not receive this imagination task.

Next, participants were asked to evaluate two different package designs for a new snack from the Japanese brand Tohato (Huang et al., 2019). The high-anthropomorphic snack was presented in first-person language with the original humanized package, while the low-anthropomorphic snack was presented in third-person language with neutral graphic elements (see appendix). Participants indicated their relative preference on an 8-point scale, with a higher number meaning higher preference for anthropomorphic products. We also measured the anthropomorphic perception of the package designs as in previous studies. Finally, we recorded the participants' demographic information like age, gender, and present residence.

\subsubsection{Results}

Manipulation check The results from the paired-samples t-test showed that the anthropomorphic perception of package designs $\left(M_{\text {high-anthropomorphic }}=5.80\right.$, $S D=1.02, M_{\text {low-anthropomorphic }}=1.74, S D=1.17, t(256)=43.15, p<0.001, d=3.70$ ) was manipulated successfully.

Product preference Following Leung et al. (2018, Study 6), we first estimated a regression model with the three conditions, the mean-centered local severity index, and their interactions as predictors. Two dummy variables were generated. The first one was the contagious disease cue without a reminder of quarantine (contagious disease cue without a reminder of quarantine condition $=1$, otherwise $=0$ ), abbreviated as contagious disease cue. The second one was the contagious disease cue with a reminder of quarantine (contagious disease cue with a reminder of quarantine condition $=1$, otherwise $=0$ ). The control condition served as the baseline, and the dependent variable was product preference. The results indicated only a significant interaction between local severity and contagious disease cue $(b=-0.013, t=-2.61$, $p=0.010$ ).

As predicted, higher severity corresponded to lower preference for anthropomorphic products in the contagious disease cue without a reminder of quarantine condition $(b=-0.010, t=-2.73, p=0.008)$, but the negative association between local severity and preference for anthropomorphic products did not appear in the control condition $(b=0.003, t=0.72, p=0.47)$ nor in the contagious disease cue with a reminder of quarantine condition $(b=-0.002, t=-0.74, p=0.46)$. Further regression analyses and floodlight analyses helped contrast the effect of local severity on preference across conditions (see Fig. 3). 


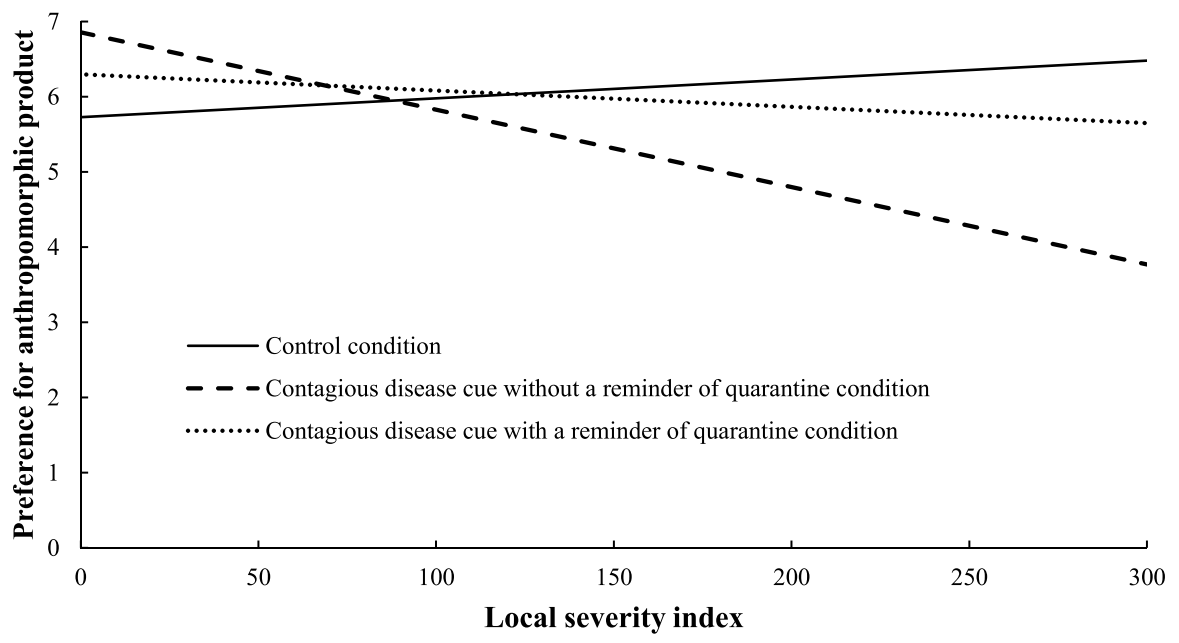

Fig. 3 Impact of contagious disease cues on preference for anthropomorphic product (Study 4)

Control versus contagious disease cue without a reminder of quarantine condition The interaction between local severity and condition $(b=-0.013, t=-2.49$, $p=0.014$ ) was significant. Floodlight analysis using the Johnson-Neyman technique (Hayes \& Matthes, 2009) revealed that for participants in regions with a severity score at 198.83 or above, contagious disease cues significantly decreased preference for anthropomorphic products $(b=-1.42, S E=0.72)$. Conversely, for participants in regions with a severity score below 198.83, the contagious disease cues effect was attenuated, supporting $\mathrm{H} 4$. Interestingly, it is reversed only when the severity score was very low, that is, at 12.64 or below $(b=0.97, S E=0.49)$. We further found that these participants (7.00\%) were from Australia (5.06\%), New Zealand (1.56\%), and Israel $(0.39 \%)$. The low population density of Australia and New Zealand not only indicates that new cases were very dispersed, but also makes interpersonal interactions highly valued (Countryaah, 2021). We believe the joint effect could reverse the contagious disease effect. For Israel, the second-highest full vaccination rate in the world may partially explain the reversed effect as vaccination reduces the perceived vulnerability (Hart, 2021).

Control versus contagious disease cue with a reminder of quarantine condition No significant effect was found in this contrast ( $p$ s $>0.30$ ), indicating the negative effect of contagious disease cues in high-severity regions and the positive effect of contagious disease cues in very-low-severity regions were all attenuated by the reminder. For high-severity regions, this might be because the manipulation instructed participants to imagine they had already been in contact with COVID-19 patients, thus reducing the utility of social withdrawal. By contrast, the low infection proportion might lead participants in very-low-severity regions to believe that COVID-19 is not a significant threat. However, the reminder broke this belief, thus increasing 
perceived vulnerability, which undermined the positive effect of contagious disease cues.

Contagious disease cue without versus with a reminder of quarantine condition We found a significant main effect of local severity $(b=-0.010, t=-2.91, p=0.004)$, but the interaction between local severity and condition was marginally significant $(b=0.008, t=1.72, p=0.087)$. However, the results of the floodlight analysis revealed that there were no statistical significance transition points within the observed range of severity score.

\section{General discussion}

Across four empirical studies, our findings consistently suggest that contagious disease cues will reduce consumer preference for anthropomorphic products, and this effect is mediated by social withdrawal. Furthermore, this detrimental effect will be alleviated for products in digital format or for consumers in regions with low local severity of the contagious disease.

This research contributes to the existing literature in multiple ways. First, this paper extends the anthropomorphism literature by identifying contagious disease cues as a novel factor that could significantly shift preference for anthropomorphic products (Puzakova \& Aggarwal, 2018). Additionally, our research introduces a new scenario in which anthropomorphism could backfire (Puzakova \& Kwak, 2017). Second, though the COVID-19 pandemic has received considerable attention, few articles have focused on the impact of contagious diseases on consumers' product preferences (Galoni et al., 2020; Huang \& Sengupta, 2020). Our research advances the extant literature on contagious diseases by extending its effect to the product preference domain. Additionally, it is interesting to note that, in our research, the quarantine reminder had different effects on preference for anthropomorphic products according to local severity. Future studies could systematically explore this issue.

Our findings also provide meaningful implications for marketing practices. We suggest that, to avoid negative consequences, marketers should be cautious about using anthropomorphism strategies during an outbreak or when promoting contagious disease-related products. More importantly, marketers could take the target consumers' location and product format into consideration when adopting anthropomorphism strategies.

\section{Appendix}

Manipulations of anthropomorphism in Studies 1-4. 
Low-anthropomorphic products

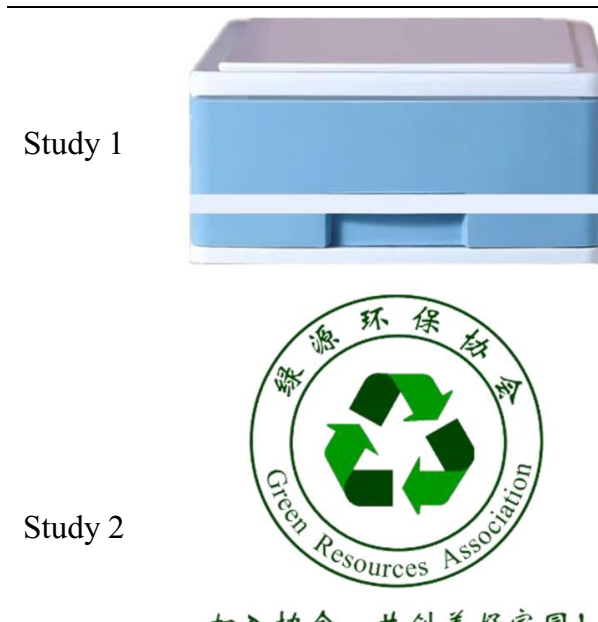

加入协会，共创美好家园!

(English meaning: Join the association to build a beautiful homeland!)

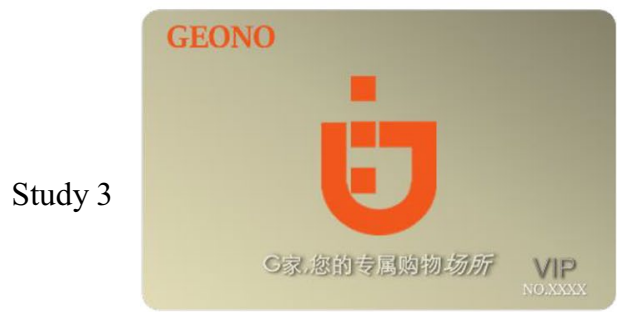

(English meaning: G home, your exclusive shopping place)

Study 4

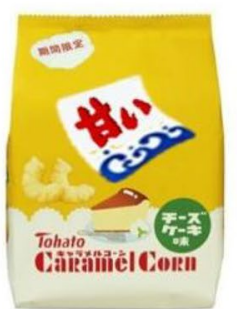

Corn snack Altius
High-anthropomorphic products
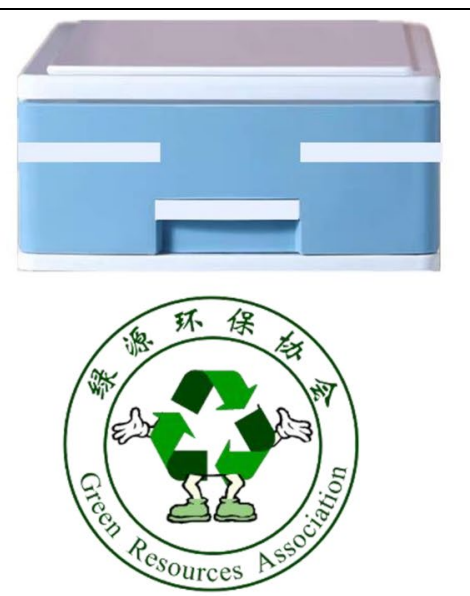

与我一起, 共创美好家园!

(English meaning: Join me to build a beautiful homeland!)

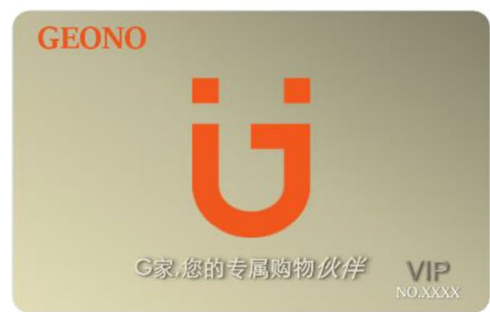

(English meaning: G home, your exclusive shopping partner)

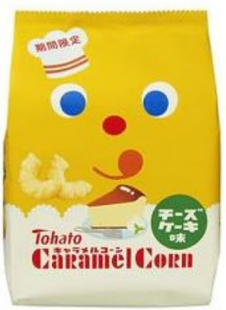

Hello! My name is Altius! 
Supplementary Information The online version contains supplementary material available at https://doi. org/10.1007/s11002-022-09614-x.

Authors' contributions Ying Ding contributed to the research conception and study design. Data collection and preliminary data analysis were performed by Sunxu Xu under the supervision of Ying Ding. The two authors discussed the data and results in many occasions. The first draft of the manuscript was written by both authors. Ying Ding commented on previous versions of the manuscript and both authors worked together to revise and finalize the manuscript.

Funding This research was supported by the National Natural Science Foundation of China research grant (No.71872173) awarded to the first author.

Data availability We have uploaded all the datasets associated with our paper onto Dropbox and we are very happy to provide any of them upon request.

Code availability Not applicable.

\section{Declarations}

Ethics approval The study design was approved by the appropriate ethics review board.

Consent to participate The participants in all the studies provided informed consent.

Consent for publication Not applicable.

Conflicts of interest The authors have no relevant financial or non-financial interests to disclose.

\section{References}

Aggarwal, P., \& McGill, A. L. (2007). Is that car smiling at me? Schema congruity as a basis for evaluating anthropomorphized products. Journal of Consumer Research, 34(4), 468-479.

Baum, A., \& Koman, S. (1976). Differential response to anticipated crowding: Psychological effects of social and spatial density. Journal of Personality and Social Psychology, 34(3), 526-536.

Belk, R. W. (2013). Extended self in a digital world. Journal of Consumer Research, 40(3), 477-500.

Boeuf, B. (2020). Boys do not cry: The negative effects of brand masculinity on brand emotions. Marketing Letters, 31(2), 247-264.

Chen, R. P., Wan, E. W., \& Levy, E. (2017). The effect of social exclusion on consumer preference for anthropomorphized brands. Journal of Consumer Psychology, 27(1), 23-34.

Countryaah. (2021). Oceania Population. Retrieved from https://www.countryaah.com/oceania-popul ation/ Accessed 5 October 2021.

Epley, N., Waytz, A., \& Cacioppo, J. T. (2007). On seeing human: A three-factor theory of anthropomorphism. Psychological Review, 114(4), 864-886.

Fournier, S. (1998). Consumers and their brands: Developing relationship theory in consumer research. Journal of Consumer Research, 24(4), 343-373.

Galoni, C., Carpenter, G. S., \& Rao, H. (2020). Disgusted and afraid: Consumer choices under the threat of contagious disease. Journal of Consumer Research, 47(3), 373-392.

Hart, R. (2021). Covid Surges In 4 Of 5 Most Vaccinated Countries-Here's Why The U.S. Should Worry. Retrieved from https://www.forbes.com/sites/roberthart/2021/05/11/covid-surges-in-4-of-5worlds-most-vaccinated-countries-heres-why-the-us-should-worry/?sh=11dddef8d677---11-may2021. Accessed 5 October 2021.

Hayes, A. F. (2013). Introduction to mediation, moderation, and conditional process analysis: A regression-based approach. The Guilford Press.

Hayes, A. F., \& Matthes, J. (2009). Computational procedures for probing interactions in OLS and logistic regression: SPSS and SAS implementations. Behavior Research Methods, 41(3), 924-936. 
Huang, F., Wong, V. C., \& Wan, E. W. (2019). The influence of product anthropomorphism on comparative judgment. Journal of Consumer Research, 46(5), 936-955.

Huang, Y., \& Sengupta, J. (2020). The influence of disease cues on preference for typical versus atypical products. Journal of Consumer Research, 47(3), 393-411.

Huang, Z., Huang, X., \& Jiang, Y. (2018). The impact of death-related media information on consumer value orientation and scope sensitivity. Journal of Marketing Research, 55(3), 432-445.

Kim, H.-Y., \& McGill, A. L. (2018). Minions for the rich? Financial status changes how consumers see products with anthropomorphic features. Journal of Consumer Research, 45(2), 429-450.

Kim, S., \& McGill, A. L. (2011). Gaming with Mr. Slot or gaming the slot machine? Power, anthropomorphism, and risk perception. Journal of Consumer Research, 38(1), 94-107.

Leary, M. R., Herbst, K. C., \& Mccrary, F. (2003). Finding pleasure in solitary activities: Desire for aloneness or disinterest in social contact? Personality and Individual Differences, 35(1), 59-68.

Leung, E., Paolacci, G., \& Puntoni, S. (2018). Man versus machine: Resisting automation in identitybased consumer behavior. Journal of Marketing Research, 55(6), 818-831.

Liu, J., \& Smeesters, D. (2010). Have you seen the news today? The effect of death-related media context. Journal of Marketing Research, 47(2), 251-262.

May, F., \& Irmak, C. (2014). Licensing indulgence in the present by distorting memories of past behavior. Journal of Consumer Research, 41(3), 624-641.

Mortensen, C. R., Becker, D. V., Ackerman, J. M., Neuberg, S. L., \& Kenrick, D. T. (2010). Infection breeds reticence: The effects of disease salience on self-perceptions of personality and behavioral avoidance tendencies. Psychological Science, 21(3), 440-447.

Puzakova, M., \& Aggarwal, P. (2018). Brands as rivals: Consumer pursuit of distinctiveness and the role of brand anthropomorphism. Journal of Consumer Research, 45(4), 869-888.

Puzakova, M., \& Kwak, H. (2017). Should anthropomorphized brands engage customers? The impact of social crowding on brand preferences. Journal of Marketing, 81(6), 99-115.

Schaller, M., \& Murray, D. R. (2008). Pathogens, personality, and culture: Disease prevalence predicts worldwide variability in sociosexuality, extraversion, and openness to experience. Journal of Personality and Social Psychology, 95(1), 212-221.

Sheth, J. (2020). Impact of Covid-19 on consumer behavior: Will the old habits return or die? Journal of Business Research, 117, 280-283.

WHO. (2021). Weekly epidemiological update on COVID-19-11 May 2021. Retrieved from https:// www.who.int/publications/m/item/weekly-epidemiological-update-on-covid-19---11-may-2021. Accessed 15 May 2021.

Publisher's note Springer Nature remains neutral with regard to jurisdictional claims in published maps and institutional affiliations. 